Supporting information

\title{
Semiempirical Model for the Solubility of Rare Earth Oxides in
}

\section{Molten Fluorides}

\author{
Xiaoling Guo ${ }^{\mathrm{a} *}$, Zhi Sun ${ }^{\mathrm{a}, \mathrm{b} *}$, Jilt Sietsma ${ }^{\mathrm{a}}$, Yongxiang Yang ${ }^{\mathrm{a}}$ \\ ${ }^{a}$ Department of Materials Science and Engineering, Delft University of Technology \\ Mekelweg 2, 2628 CD Delft, The Netherlands \\ ${ }^{b}$ National Engineering Laboratory for Hydrometallurgical Cleaner Production Technology, \\ Institute of Process Engineering, Chinese Academy of Sciences, Beijing 100190, China
} *Corresponding authors: Xiaoling Guo (shelley_goo@msn.com; x.guo-1@tudelft.nl) and Zhi Sun (zhisun@126.com) 
Table S1 Solubility of rare earth oxides in fluoride melts

\begin{tabular}{|c|c|c|c|c|c|}
\hline REO & Melt & Temperature, $\mathrm{K}$ & $\begin{array}{c}\text { Solubility, } \\
\text { mol.\% }\end{array}$ & $\begin{array}{l}\text { solubility, } \\
\text { wt. } \%\end{array}$ & Reference \\
\hline \multirow{39}{*}{$\mathrm{Y}_{2} \mathrm{O}_{3}$} & \multirow{8}{*}{$25 \mathrm{YF}_{3}-75 \mathrm{LiF}$} & 1083 & 0.11 & 0.45 & \multirow{29}{*}{1} \\
\hline & & 1104 & 0.12 & 0.50 & \\
\hline & & 1125 & 0.15 & 0.59 & \\
\hline & & 1155 & 0.20 & 0.80 & \\
\hline & & 1172 & 0.20 & 0.80 & \\
\hline & & 1273 & 0.41 & 1.65 & \\
\hline & & 1178 & 0.22 & 0.88 & \\
\hline & & 1270 & 0.39 & 1.56 & \\
\hline & \multirow{9}{*}{$50 \mathrm{YF}_{3}-50 \mathrm{LiF}$} & 1175 & 0.46 & 1.19 & \\
\hline & & 1202 & 0.54 & 1.41 & \\
\hline & & 1216 & 0.58 & 1.51 & \\
\hline & & 1237 & 0.62 & 1.61 & \\
\hline & & 1247 & 0.69 & 1.78 & \\
\hline & & 1261 & 0.72 & 1.88 & \\
\hline & & 1273 & 0.77 & 1.98 & \\
\hline & & 1278 & 0.77 & 2.00 & \\
\hline & & 1282 & 0.81 & 2.09 & \\
\hline & $35 \mathrm{YF}_{3}-65 \mathrm{LiF}$ & 1273 & 0.51 & 1.69 & \\
\hline & $65 \mathrm{YF}_{3}-35 \mathrm{LiF}$ & 1273 & 1.03 & 2.20 & \\
\hline & \multirow{5}{*}{$50 \mathrm{YF}_{3}-50 \mathrm{NaF}$} & 1137 & 0.17 & 0.40 & \\
\hline & & 1175 & 0.21 & 0.50 & \\
\hline & & 1206 & 0.25 & 0.60 & \\
\hline & & 1229 & 0.29 & 0.70 & \\
\hline & & 1277 & 0.42 & 1.00 & \\
\hline & \multirow{5}{*}{$25 \mathrm{YF}_{3}-75 \mathrm{NaF}$} & 1186 & 0.11 & 0.35 & \\
\hline & & 1220 & 0.12 & 0.40 & \\
\hline & & 1231 & 0.14 & 0.45 & \\
\hline & & 1251 & 0.15 & 0.50 & \\
\hline & & 1282 & 0.18 & 0.60 & \\
\hline & $81 \mathrm{LiF}-15 \mathrm{MgF}_{2}-4 \mathrm{BaF}_{2}$ & 1273 & 1.16 & 6.55 & 2 \\
\hline & \multirow{7}{*}{$20 \mathrm{YF}_{3}-80 \mathrm{LiF}$} & 998 & 0.26 & 1.17 & \multirow{9}{*}{3} \\
\hline & & 1023 & 0.28 & 1.25 & \\
\hline & & 1073 & 0.33 & 1.48 & \\
\hline & & 1148 & 0.42 & 1.87 & \\
\hline & & 1193 & 0.47 & 2.09 & \\
\hline & & 1223 & 0.53 & 2.35 & \\
\hline & & 1273 & 0.65 & 2.87 & \\
\hline & \multirow{2}{*}{$25 \mathrm{YF}_{3}-75 \mathrm{LiF}$} & 1023 & 0.32 & 1.28 & \\
\hline & & 1098 & 0.41 & 1.63 & \\
\hline
\end{tabular}




\begin{tabular}{|c|c|c|c|c|c|}
\hline REO & Melt & Temperature, $\mathrm{K}$ & $\begin{array}{c}\text { Solubility, } \\
\text { mol. } \%\end{array}$ & $\begin{array}{c}\text { solubility, } \\
\text { wt.\% }\end{array}$ & Reference \\
\hline & & 1123 & 0.45 & 1.79 & \\
\hline & & 1173 & 0.53 & 2.11 & \\
\hline & & 1273 & 0.75 & 2.96 & \\
\hline & \multirow{6}{*}{$40 \mathrm{YF}_{3}-60 \mathrm{LiF}$} & 1023 & 0.57 & 1.72 & \\
\hline & & 1073 & 0.71 & 2.14 & \\
\hline & & 1098 & 0.75 & 2.26 & \\
\hline & & 1173 & 0.98 & 2.93 & \\
\hline & & 1223 & 1.18 & 3.52 & \\
\hline & & 1273 & 1.66 & 4.90 & \\
\hline & \multirow{3}{*}{$50 \mathrm{YF}_{3}-50 \mathrm{LiF}$} & 1173 & 1.45 & 3.72 & \\
\hline & & 1223 & 1.74 & 4.45 & \\
\hline & & 1273 & 2.11 & 5.36 & \\
\hline \multirow{31}{*}{$\mathrm{La}_{2} \mathrm{O}_{3}$} & $41 \mathrm{LaF}_{3}-59 \mathrm{LiF}$ & 1223 & 0.78 & 2.60 & \multirow[t]{2}{*}{4} \\
\hline & $30 \mathrm{LaF}_{3}-65 \mathrm{LiF}-5 \mathrm{BaF}_{2}$ & 1223 & 0.61 & 2.30 & \\
\hline & $81 \mathrm{LiF}-15 \mathrm{MgF}_{2}-4 \mathrm{BaF}_{2}$ & 1273 & 1.76 & 13.37 & 2 \\
\hline & \multirow{2}{*}{$\mathrm{LiF}$} & 1107 & 2.50 & 24.36 & \multirow{12}{*}{5} \\
\hline & & 1123 & 3.00 & 27.98 & \\
\hline & \multirow{2}{*}{$\mathrm{NaF}$} & 1255 & 2.00 & 13.67 & \\
\hline & & 1268 & 2.50 & 16.59 & \\
\hline & \multirow{2}{*}{$\mathrm{KF}$} & 1133 & 1.50 & 7.87 & \\
\hline & & 1144 & 2.00 & 10.27 & \\
\hline & \multirow{6}{*}{ 46.5LiF-11.5NaF-42KF } & 720 & 1.50 & 10.73 & \\
\hline & & 727 & 2.00 & 13.87 & \\
\hline & & 743 & 3.00 & 19.62 & \\
\hline & & 763 & 4.00 & 24.74 & \\
\hline & & 786 & 5.00 & 29.34 & \\
\hline & & 808 & 6.00 & 33.50 & \\
\hline & \multirow{3}{*}{$75 \mathrm{LiF}-25 \mathrm{AlF}_{3}$} & 1039 & 10.00 & 47.22 & \multirow{9}{*}{6} \\
\hline & & 1037 & 10.00 & 47.24 & \\
\hline & & 1028 & 9.51 & 45.85 & \\
\hline & \multirow{3}{*}{$75 \mathrm{NaF}-25 \mathrm{AlF}_{3}$} & 1211 & 11.51 & 44.67 & \\
\hline & & 1226 & 12.01 & 45.86 & \\
\hline & & 1251 & 12.99 & 48.11 & \\
\hline & \multirow{3}{*}{$75 \mathrm{KF}-25 \mathrm{AlF}_{3}$} & 1208 & 11.48 & 39.55 & \\
\hline & & 1225 & 11.98 & 40.72 & \\
\hline & & 1251 & 12.99 & 42.96 & \\
\hline & \multirow{5}{*}{$51 \mathrm{LiF}-49 \mathrm{ZrF}_{4}$} & 873 & 0.20 & 0.67 & \multirow{7}{*}{7} \\
\hline & & 923 & 0.27 & 0.92 & \\
\hline & & 973 & 0.37 & 1.27 & \\
\hline & & 1023 & 0.52 & 1.74 & \\
\hline & & 1073 & 0.60 & 2.03 & \\
\hline & \multirow{2}{*}{$50.5 \mathrm{NaF}-49.5 \mathrm{ZrF}_{4}$} & 873 & 0.29 & 0.91 & \\
\hline & & 923 & 0.34 & 1.07 & \\
\hline
\end{tabular}




\begin{tabular}{|c|c|c|c|c|c|}
\hline \multirow[t]{9}{*}{ REO } & \multirow[t]{4}{*}{ Melt } & Temperature, $\mathrm{K}$ & $\begin{array}{c}\text { Solubility, } \\
\text { mol. } \%\end{array}$ & $\begin{array}{c}\text { solubility, } \\
\text { wt. } \%\end{array}$ & \multirow[t]{2}{*}{ Reference } \\
\hline & & 973 & 0.38 & 1.19 & \\
\hline & & 1023 & 0.46 & 1.42 & \\
\hline & & 1073 & 0.49 & 1.52 & \\
\hline & \multirow{5}{*}{$58 \mathrm{KF}-42 \mathrm{ZrF}_{4}$} & 873 & 0.04 & 0.12 & \\
\hline & & 923 & 0.04 & 0.12 & \\
\hline & & 973 & 0.05 & 0.16 & \\
\hline & & 1023 & 0.06 & 0.19 & \\
\hline & & 1073 & 0.08 & 0.27 & \\
\hline \multirow{9}{*}{$\mathrm{CeO}_{2}$} & \multirow{2}{*}{$26 \mathrm{CeF}_{3}-66 \mathrm{LiF}-8 \mathrm{BaF}_{2}$} & 1123 & 1.01 & 2.10 & \multirow[t]{2}{*}{4} \\
\hline & & 1073 & 0.67 & 1.40 & \\
\hline & $81 \mathrm{LiF}-15 \mathrm{MgF}_{2}-4 \mathrm{BaF}_{2}$ & 1273 & 3.75 & 15.09 & 2 \\
\hline & \multirow{6}{*}{$75 \mathrm{NaF}-25 \mathrm{AlF}_{3}$} & 1278 & 1.23 & 3.91 & \multirow{6}{*}{8} \\
\hline & & 1285 & 1.19 & 3.80 & \\
\hline & & 1293 & 1.29 & 4.11 & \\
\hline & & 1293 & 1.33 & 4.22 & \\
\hline & & 1293 & 1.42 & 4.52 & \\
\hline & & 1303 & 1.43 & 4.54 & \\
\hline $\mathrm{Pr}_{2} \mathrm{O}_{3}$ & $81 \mathrm{LiF}-15 \mathrm{MgF}_{2}-4 \mathrm{BaF}_{2}$ & 1273 & 0.72 & 5.94 & 2 \\
\hline \multirow{24}{*}{$\mathrm{Nd}_{2} \mathrm{O}_{3}$} & $75 \mathrm{~L}$ : $25 \mathrm{Ma}$ & 1273 & 0.96 & 8.50 & \multirow{24}{*}{9} \\
\hline & $/ J \mathrm{LiF}-2 \mathrm{JMgF} \mathrm{P}_{2}$ & 1273 & 0.14 & 1.20 & \\
\hline & $76 \mathrm{LiF}-18 \mathrm{MgF}_{2}-6 \mathrm{CaF}_{2}$ & 1273 & 0.73 & 6.50 & \\
\hline & $771 \mathrm{LiF}-14 \mathrm{MgF}_{2}-9 \mathrm{CaF}_{2}$ & 1273 & 0.62 & 5.52 & \\
\hline & $77 \mathrm{LiF}-10 \mathrm{MgF}_{2}-12 \mathrm{CaF}_{2}$ & 1273 & 0.44 & 3.92 & \\
\hline & $78 \mathrm{LiF}-6.5 \mathrm{MgF}_{2}-15.5 \mathrm{CaF}_{2}$ & 1273 & 0.28 & 2.57 & \\
\hline & $79 \mathrm{LiF}-18 \mathrm{MgF}_{2}-3 \mathrm{BaF}_{2}$ & 1273 & 0.74 & 6.39 & \\
\hline & \multirow{2}{*}{$81 \mathrm{LiF}-15 \mathrm{MgF}_{2}-4 \mathrm{BaF}_{2}$} & 1273 & 0.63 & 5.38 & \\
\hline & & 1273 & 0.70 & 5.88 & \\
\hline & $83 \mathrm{LiF}-11 \mathrm{MgF}_{2}-6 \mathrm{BaF}_{2}$ & 1273 & 0.45 & 3.76 & \\
\hline & $85 \mathrm{LiF}-7 \mathrm{MgF}_{2}-8 \mathrm{BaF}_{2}$ & 1273 & 0.26 & 2.12 & \\
\hline & $23 \mathrm{NdF}_{3}-77 \mathrm{LiF}$ & 1123 & 1.97 & 9.24 & \\
\hline & \multirow{3}{*}{$28 \mathrm{NdF}_{3}-72 \mathrm{LiF}$} & 1073 & 1.70 & 7.20 & \\
\hline & & 1123 & 1.97 & 8.30 & \\
\hline & & 1173 & 2.26 & 9.40 & \\
\hline & $34 \mathrm{NdF}_{3}-66 \mathrm{LiF}$ & 1123 & 1.96 & 7.29 & \\
\hline & $20 \mathrm{NdF}_{3}-76 \mathrm{LiF}-4 \mathrm{BaF}_{2}$ & 1123 & 1.94 & 9.14 & \\
\hline & \multirow{3}{*}{$21 \mathrm{NdF}_{3}-77 \mathrm{LiF}-2 \mathrm{BaF}_{2}$} & 1073 & 1.80 & 8.51 & \\
\hline & & 1123 & 1.96 & 9.19 & \\
\hline & & 1173 & 2.11 & 9.88 & \\
\hline & \multirow{3}{*}{$24 \mathrm{NdF}_{3}-72 \mathrm{LiF}-4 \mathrm{BaF}_{2}$} & 1073 & 1.90 & 8.07 & \\
\hline & & 1123 & 2.15 & 9.04 & \\
\hline & & 1173 & 2.42 & 10.10 & \\
\hline & $26 \mathrm{NdF}_{3}-72 \mathrm{LiF}-2 \mathrm{BaF}_{2}$ & 1073 & 1.79 & 7.59 & \\
\hline
\end{tabular}




\begin{tabular}{|c|c|c|c|c|c|}
\hline REO & Melt & Temperature, $\mathrm{K}$ & $\begin{array}{l}\text { Solubility, } \\
\text { mol. } \%\end{array}$ & $\begin{array}{l}\text { solubility, } \\
\text { wt. } \%\end{array}$ & Reference \\
\hline & & 1123 & 2.05 & 8.61 & \multirow{9}{*}{10} \\
\hline & & 1173 & 2.32 & 9.67 & \\
\hline & $30 \mathrm{NdF}_{3}-66 \mathrm{LiF}-5 \mathrm{BaF}_{2}$ & 1123 & 2.42 & 8.93 & \\
\hline & \multirow{3}{*}{$32 \mathrm{NdF}_{3}-66 \mathrm{LiF}-2 \mathrm{BaF}_{2}$} & 1073 & 1.78 & 6.67 & \\
\hline & & 1123 & 2.17 & 8.06 & \\
\hline & & 1173 & 2.58 & 9.47 & \\
\hline & $5 \mathrm{NdF}_{3}-76 \mathrm{LiF}-19 \mathrm{CaF}_{2}$ & 1303 & 0.40 & 2.93 & \\
\hline & $10 \mathrm{NdF}_{3}-72 \mathrm{LiF}-18 \mathrm{CaF}_{2}$ & 1303 & 0.51 & 3.15 & \\
\hline & $20 \mathrm{NdF}_{3}-64 \mathrm{LiF}-16 \mathrm{CaF}_{2}$ & 1303 & 0.83 & 3.92 & \\
\hline & \multirow{4}{*}{$15 \mathrm{NdF}_{3}-85 \mathrm{LiF}$} & 1073 & 0.08 & 0.54 & \multirow{17}{*}{11} \\
\hline & & 1123 & 0.12 & 0.75 & \\
\hline & & 1133 & 0.13 & 0.82 & \\
\hline & & 1173 & 0.15 & 0.96 & \\
\hline & \multirow{4}{*}{$23.1 \mathrm{NdF}_{3}-76.9 \mathrm{LiF}$} & 1023 & 0.13 & 0.68 & \\
\hline & & 1073 & 0.16 & 0.80 & \\
\hline & & 1123 & 0.18 & 0.92 & \\
\hline & & 1173 & 0.22 & 1.11 & \\
\hline & \multirow{2}{*}{$30 \mathrm{NdF}_{3}-70 \mathrm{LiF}$} & 1133 & 0.34 & 1.45 & \\
\hline & & 1173 & 0.38 & 1.61 & \\
\hline & \multirow{2}{*}{$15 \mathrm{NdF}_{3}-73.8 \mathrm{LiF}-11.2 \mathrm{MgF}_{2}$} & 1073 & 0.12 & 0.71 & \\
\hline & & 1133 & 0.16 & 0.95 & \\
\hline & \multirow{2}{*}{$15 \mathrm{NdF}_{3}-79.4 \mathrm{LiF}-5.6 \mathrm{MgF}_{2}$} & 1073 & 0.12 & 0.74 & \\
\hline & & 1133 & 0.15 & 0.92 & \\
\hline & $30 \mathrm{NdF}_{3}-59.2 \mathrm{LiF}-10.8 \mathrm{MgF}_{2}$ & 1133 & 0.30 & 1.21 & \\
\hline & $30 \mathrm{NdF}_{3}-67.3 \mathrm{LiF}-2.7 \mathrm{MgF}_{2}$ & 1133 & 0.33 & 1.38 & \\
\hline & $30 \mathrm{NdF}_{3}-69 \mathrm{LiF}-1 \mathrm{MgF}_{2}$ & 1133 & 0.34 & 1.43 & \\
\hline & \multirow{3}{*}{$42 \mathrm{NdF}_{3}-58 \mathrm{LiF}$} & 1323 & 1.00 & 3.28 & \multirow{9}{*}{12} \\
\hline & & 1423 & 1.39 & 4.54 & \\
\hline & & 1373 & 1.18 & 3.88 & \\
\hline & \multirow{3}{*}{$50 \mathrm{NdF}_{3}-50 \mathrm{LiF}$} & 1323 & 1.29 & 3.74 & \\
\hline & & 1373 & 1.46 & 4.23 & \\
\hline & & 1423 & 1.64 & 4.71 & \\
\hline & \multirow{3}{*}{$60 \mathrm{NdF}_{3}-40 \mathrm{LiF}$} & 1323 & 1.67 & 4.20 & \\
\hline & & 1373 & 1.85 & 4.62 & \\
\hline & & 1423 & 1.99 & 4.97 & \\
\hline \multirow{7}{*}{$\mathrm{Sm}_{2} \mathrm{O}_{3}$} & $81 \mathrm{LiF}-15 \mathrm{MgF}_{2}-4 \mathrm{BaF}_{2}$ & 1273 & 0.49 & 4.35 & 2 \\
\hline & & 873 & 0.20 & 0.73 & \multirow{6}{*}{7} \\
\hline & & 923 & 0.26 & 0.95 & \\
\hline & $51 \mathrm{LiF}-49 \mathrm{ZrF}_{4}$ & 973 & 0.39 & 1.40 & \\
\hline & & 1023 & 0.57 & 2.05 & \\
\hline & & 1073 & 0.65 & 2.35 & \\
\hline & $50.5 \mathrm{NaF}-49.5 \mathrm{ZrF}_{4}$ & 873 & 0.27 & 0.91 & \\
\hline
\end{tabular}




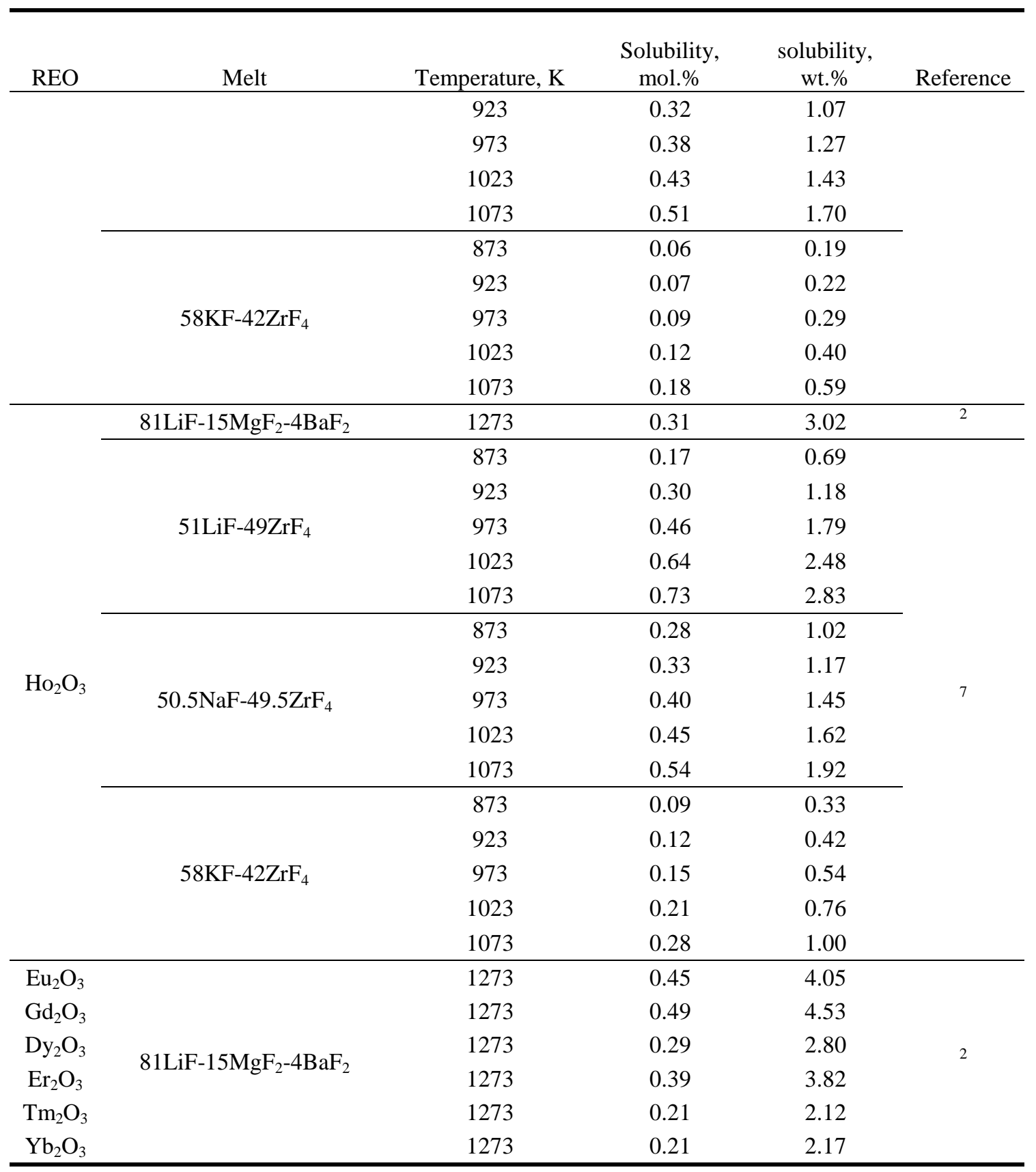

\section{References}

1. Bratland, D. On the Possible Electrowinning of Yt-Al Alloys. The Solubility of Yttria and of Alumina in Molten Mixtures of Yttrium Fluoride and Lithium Fluoride. Light Metals 1976. 1976, 1, 183-201. 
2. Du, S.; Wu, M.; Du, F.; Liu, Y. Solubility of Rare Earth Oxides in Alkali and Alkali-Earth Metal Fluoride Melts. Chin. Rare Earths 1987, 8 (2), 59-62.

3. (a) Morrice, E.; Reddy, R. G. In Solubility and Activity Coefficient of $\mathrm{Y}_{2} \mathrm{O}_{3}$ in Fluoride Melts, Rare Earths, Extraction, Preparation and Applications sponsored, Las Vegas, Nevada, Bautista, R. G.; Wong, M. M., Eds. TMS: Las Vegas, Nevada, 1989; pp 377-388; (b) Reddy, R. G.; Kumar, S. G. Solubility and Thermodynamic Properties of $\mathrm{Y}_{2} \mathrm{O}_{3}$ in LiF- $\mathrm{YF}_{3}$ Melts. Metall. Mater. Trans. B-Proc. Metall. Mater. Proc. Sci. 1994, 25 (1), 91-96.

4. Porter, B. B. E. A. Determination of Oxide Solubility in Molten Fluorides; U.S. Dept. of the Interior, Bureau of Mines: Washington, DC, 1961.

5. Ambrová, M.; Jurišová, J.; Danielik, V.; Gabčová, J. On the Solubility of Lanthanum Oxide in Molten Alkali Fluorides. J. Therm. Anal. Calorim. 2008, 91 (2), 569-573.

6. Ambrová, M.; Jurišová, J. Solubilities of Lanthanum Oxide in Fluoride Melts Part I. Solubility in $\mathrm{M}_{3} \mathrm{AlF}_{6}(\mathrm{M}=\mathrm{Li}, \mathrm{Na}, \mathrm{K})$. Thermochim. Acta 2006, 443 (1), 105-108.

7. Pshenichny, R. N.; Omelchuk, A. A. Interaction of Rare-Earth Oxides with Binary Molten Mixtures of Zirconium and Alkali Metal Fluorides. Russ. J. Inorg. Chem. 2012, 57 (1), 115-119.

8. Dewing, E. W.; Haarberg, G. R.; Rolseth, S.; Ronne, L.; Thonstad, J.; Aalberg, N. The Chemistry of Solutions of $\mathrm{CeO}_{2}$ in Cryolite Melts. Metall. Mater. Trans. BProc. Metall. Mater. Proc. Sci. 1995, 26 (1), 81-86.

9. Wu, W.; Sun, J.; Hai, L.; Gao, H. $\mathrm{Nd}_{2} \mathrm{O}_{3}$ Solubility in Fluoride Melt. Chin. Rare Earths 1991, 12 (3), 34-37.

10. Murphy, J.; Dysinger, D.; Chambers, M. Electrowinning Neodymium Metal from Chloride and Oxide-Fluoride Electrolytes. In Trace and Reactive Metals: Processing and Technology, Reddy, R. G., Ed. 1995; pp 137-151. 
11. Stefanidaki, E.; Photiadis, G. M.; Kontoyannis, C. G.; Vik, A. F.; Ostvold, T. Oxide Solubility and Raman Spectra of $\mathrm{NdF}_{3}-\mathrm{LiF}-\mathrm{KF}-\mathrm{MgF}_{2}-\mathrm{Nd}_{2} \mathrm{O}_{3}$ Melts. J. Chem. Soc., Dalton Trans. 2002, (11), 2302-2307.

12. $\mathrm{Hu}, \mathrm{X}$. Study on Ionic Structure and Its Application of $\mathrm{NdF}_{3}-\mathrm{LiF}_{-} \mathrm{Nd}_{2} \mathrm{O}_{3}$ System Melts. Doctoral dissertation, Northeastern University, 2008. 